\title{
A Modified Matched Filter With Double-Sided Thresholding for Screening Proliferative Diabetic Retinopathy
}

\author{
Lei Zhang, Member, IEEE, Qin Li, Jane You, Member, IEEE, and David Zhang, Fellow, IEEE
}

\begin{abstract}
The early diagnosis of proliferative diabetic retinopathy (PDR), a common complication of diabetes that damages the retina, is crucial to the protection of the vision of diabetes sufferers. The onset of PDR is signaled by the appearance of neovascular net. Such neovascular nets might be identified using retinal vessel extraction techniques. The commonly used matched filter methods often produce false positive detections of neovascular nets due to their proneness to detect nonline edges as well as lines. In this paper, we propose a modified matched filter for retinal vessel extraction that applies a local vessel cross-section analysis using double-sided thresholding to reduce false responses to nonline edges. Our proposed modified matched filters demonstrated higher true positive rate and lesser false detection than existing matched-filter-based schemes in vessel extraction.
\end{abstract}

Index Terms-Matched filter, proliferative diabetic retinopathy (PDR), retinal image, vessel extraction.

\section{INTRODUCTION}

I MAGES of the ocular fundus, i.e., retinal images, can assist in the diagnosis and treatment of retinal, ophthalmic, and even systemic diseases, such as diabetes, hypertension, and arteriosclerosis. A critical feature in such images is the blood vessel; so the development of an efficient algorithm for automated segmentation of blood vessels would certainly help eye-care specialists in the important task of screening large populations for vessel abnormalities. One such application is in screening for diabetic retinopathy (DR) [1]-[3]. Proliferative DR (PDR), a common complication of diabetes that damages the eye's retina, can be most effectively treated with early diagnosis. Comparing with nonproliferative DR (NPDR), one early sign of PDR is the appearance of neovascular nets, i.e., nets of new blood vessels.

Many methods have been proposed for retinal vessel extraction [1], [2], [4]-[15], but neovascular extraction is still a difficult problem. Vessel segmentation is a kind of line detection problem, and many vessel extraction algorithms are based on line detection techniques [16]-[20]. There are two

Manuscript received January 29, 2008. First published April 21, 2009; current version published July 6, 2009. This work was supported by the Hong Kong Government (RGC), Natural Science Foundation of China (NSFC), under Grant 60620160097, by the 863 Fund of China under Grant 2006AA01Z193, and by the Hong Kong Polytechnic University.

L. Zhang, J. You, and D. Zhang are with the Biometric Research Center, Department of Computing, the Hong Kong Polytechnic University, Kowloon, Hong Kong (e-mail: cslzhang @ comp.polyu.edu.hk).

Q. Li is with the Biometric Research Center, Department of Computing, the Hong Kong Polytechnic University, Kowloon, Hong Kong, and also with the Henan Provincial Key Laboratory on Information Networks, Zhengzhou University, 450001 Zhengzhou, China.

Color versions of one or more of the figures in this paper are available online at http://ieeexplore.ieee.org.

Digital Object Identifier 10.1109/TITB.2008.2007201 usual steps in a vessel segmentation scheme, enhancement and classification, with some methods leading directly to classification. Vessel enhancement is usually implemented locally by using a window centered on the pixel to be enhanced. The matched filter was first proposed to detect retinal vessels in [4], and it is now widely used. Other commonly used enhancement algorithms have applied Gabor filters [2], [6], mathematical morphology filters [8], [10], and Hessian-based methods [11], [12]. Vessel enhancement involves classifying pixels as either vessel or nonvessel pixels. The easiest way is to find an optimal threshold to classify the pixels according to their intensities, and the result can then be improved at a price of more computation. A threshold probing technique was proposed in [5] to improve the accuracy of matched filters by analyzing the region-based attributes of the vessel network structure. A multithreshold probing algorithm was applied directly to the original retinal images for classification in [9]. Alternatives to these are the tracking-based methods that utilize not only the intensity information, but also the geometrical and topological information [13]-[15] and supervised learning methods [1], [2].

Of these methods, matched filter with threshold probing [4], [5] has demonstrated good performance with relatively low complexity. This matched filter makes use of the fact that the cross section of a retinal vessel is Gaussian-shaped. This allows a Gaussian-shaped filter to be used to match the vessel. If a vessel has a width that matches the scale of the filter, there will be a strong response. Another advantage of the Gaussianshaped matched filter is that it can reduce noise. Unfortunately, like other line detectors, such as the second-order derivative of Gaussian [17] and Gabor filters [2], [6], the matched filters respond not only to vessels but also to nonvessel edges. This means that if we are detecting the dark lines in an image, the edges of bright blobs (e.g., bright lesions and the optic disk) will be incorrectly detected as lines. Further, several lesions may together be detected as a neovascular net, and this would lead to false diagnosis of PDR.

A number of methods have sought to eliminate the false positives caused by lesions. Threshold probing using the vessel structural information was applied in [5]. A vessel detection scheme that uses the first-order derivative of Gaussian and modified top-hat operation was proposed in [8]. This does suppress the false detections caused by bright blobs, but the preprocessing of small line enhancement and the later morphological reconstruction has the potential to produce more false detections than schemes without preprocessing. 
In this paper, we propose a modified matched filter that suppresses false detections caused by bright blobs. While the traditional matched filter methods for detecting vessels remove the background by subtracting the local mean from the response and then thresholding, our approach first applies Gaussian filters to enhance the retinal image and then uses a double-sided thresholding to analyze the local structures of filtered outputs. This modified matched filter approach therefore significantly reduces the false detection of vessels because it avoids responding to nonline edges.

The rest of this paper is organized as follows. Section II introduces the problem of matched filter in vessel enhancement. Section III presents the modified matched filter using local double-sided thresholding. Experimental results are shown in Section IV and conclusions are presented in Section V.

\section{MAtched Filter AND ITS PROBlems IN VESSEL EXTRACTION}

The use of a matched filter for retinal vessel detection was first proposed in [4]. It makes use of the fact that the cross section of the vessels in a retinal image has the shape of a Gaussian function. Thus, a Gaussian-shaped filter can be used to "match" the vessels. Mathematically, the matched filter can be described as

$g(x, y)=\exp \left(\frac{-x^{2}}{\sigma^{2}}\right)-m, \quad$ for $|x| \leq 3 \sigma, \quad|y| \leq \frac{L}{2}$

where $\sigma$ represents the scale of the filter, $m=$ $\left(\int_{-3 \sigma}^{3 \sigma} \exp \left(-x^{2} / \sigma^{2}\right) d x\right) / 6 \sigma$ is used to normalize the mean value of the filter to 0 so that the smooth background can be removed after filtering, and $L$ is the length of the neighborhood along $y$-axis to smooth noise. In implementation, $g(x, y)$ will be rotated to detect the vessels of different orientations. The rotation of $g(x, y)$ with angle $\phi$ is

$$
\left\{\begin{array}{l}
g^{\phi}\left(x^{\prime}, y^{\prime}\right)=g(x, y) \\
x^{\prime}=x \cos \phi+y \sin \phi \\
y^{\prime}=y \cos \phi-x \sin \phi
\end{array}\right.
$$

One problem of the matched filter is that it responds not only to lines but also to nonline edges. This problem also exists in other line detectors, such as the Gabor filter and the secondorder derivative of Gaussian. Fig. 1 illustrates this by showing the responses of different filters to a Gaussian function (i.e., the cross section of a vessel) and an ideal step edge. We can see that all the three filters have strong responses to both the line cross section and the nonline ideal step edge. Fig. 2 shows the response of the matched filter to an NPDR retinal image. We can clearly see the strong responses to the vessels, as well as the edges of the bright lesions. After thresholding, both the vessels and the edges of lesions will be detected. It is of high demand to develop a new vessel detection scheme that can effectively distinguish vessels and lesions.
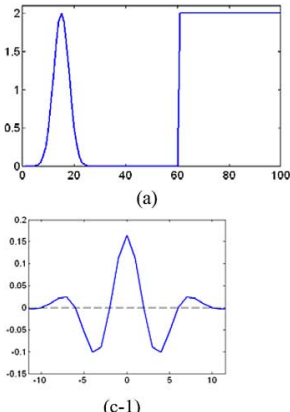

(c-1)

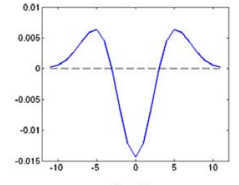

(b-1)

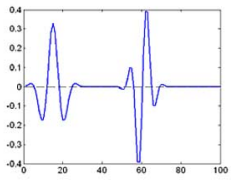

(c-2)

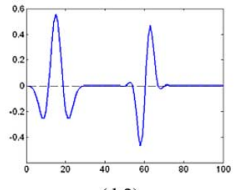

(d-2)

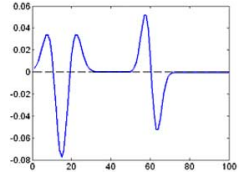

(b-2)

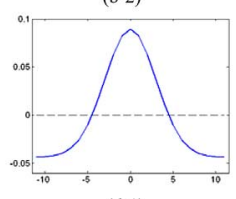

(d-1)
Fig. 1. Responses of different line detectors to a Gaussian line cross section and an ideal step edge. (a) Gaussian line cross section and an ideal step edge. (b-1) Second-order derivative of Gaussian and (b-2) its filter response (c-1) Gabor filter and (c-2) its filter response. (d-1) Matched filter and (d-2) its filter response.

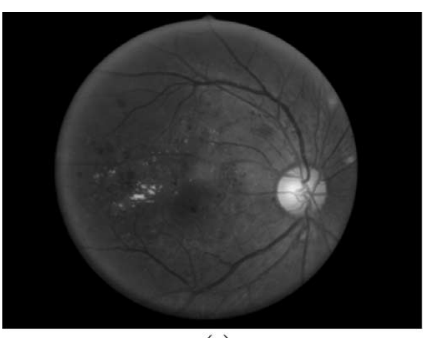

(a)

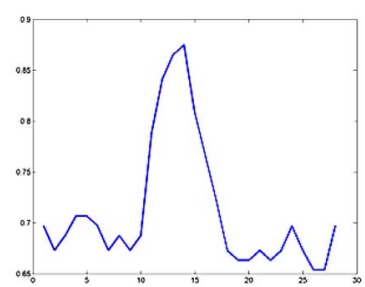

(c)

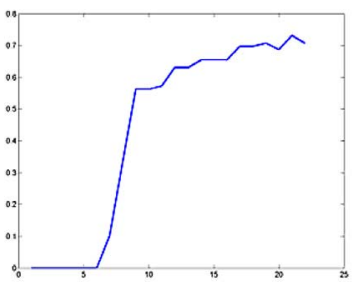

(e)

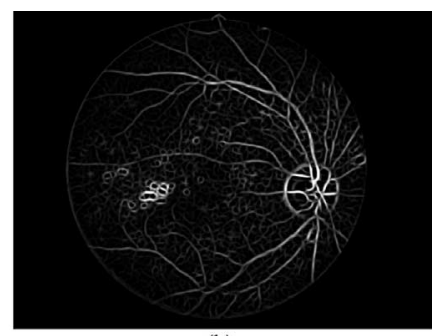

(b)

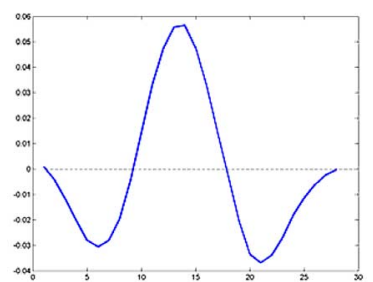

(d)

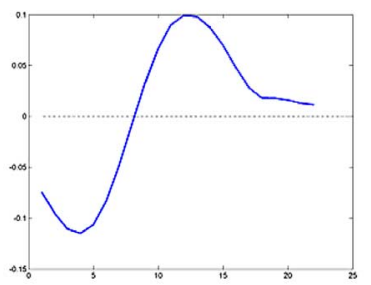

(f)
Fig. 2. (a) Retinal image with NPDR. (b) Matched filter response to the image in (a). (c) Cross section of a vessel in (a). (d) Matched filter response to (c). (e) Edge of a bright lesion in (a). (f) Matched filter response to (e).

\section{Modified MATCHED FILTER With DOUBLE-SideD THRESHOLDING}

As was seen in (1), the matched filter scheme will subtract mean value $m$ from the Gaussian function to remove the smooth background in the filtered output. It then applies a thresholding operation to detect the vessel pixels. In our approach, however, 


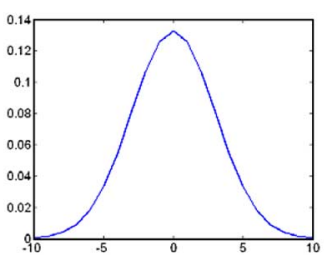

(a)

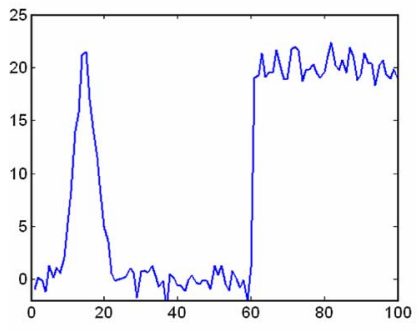

(c)

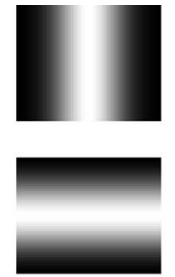

(b)

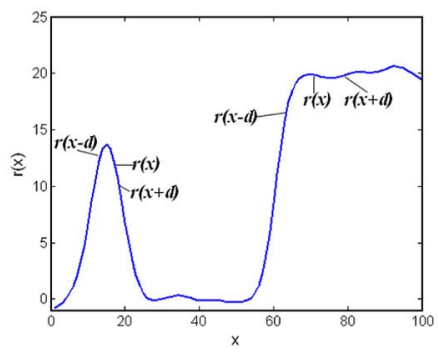

(d)
Fig. 3. Modified matched filter in (a) 1-D and (b) 2-D. (c) Gaussian function and a step edge with noise. (d) Modified matched filter's response to the signal in (c).

we do not subtract the mean from the Gaussian filter and modify the matched filter in (1) as

$$
g(x, y)=\exp \left(\frac{-x^{2}}{\sigma^{2}}\right), \quad \text { for }|x| \leq 3 \sigma, \quad|y| \leq \frac{L}{2} .
$$

Actually, the 2-D modified matched filter in (3) is a truncated Gaussian function in $x$-direction and the Gaussian function repeats in $y$-direction. Fig. 3(a) and (b) shows the 1-D cross section of the filter and the 2-D filter in four different directions. Fig. 3(c) and (d) shows a 1-D noisy signal, which contains a Gaussian function and a step edge, and its filtering response.

The modified matched filter will be applied to the complement of the original retinal image, in which the vessels are brighter than the background. The image will be filtered by $g(x, y)$ in eight directions. Each of the eight filtering responses will be thresholded to generate a vessel map. Finally, the eight vessel maps are fused through a logical OR operation. Next we introduce the proposed thresholding strategy.

After image enhancement by using the modified matched filters, the pixels need to be classified into vessel pixels and nonvessel pixels. We propose here a local double-sided thresholding scheme to segment the retinal image. To simplify the analysis, we consider the 1-D case, i.e., a 1-D signal $f(x)$ is convolved with the 1-D matched filter $g(x): r(x)=f(x) * g(x)$. Referring to Fig. 3(d), we can see that if $r(x)$ is the peak point of a Gaussian-shaped cross section of a vessel, it should be greater than both its left and right neighboring points. If $r(x)$ is from the nonvessel edges, it will not be much greater than its neighbors on both sides. Based on this observation, the vessels and nonvessel edges can be distinguished.

We define that a point $r(x)$ is a vessel point if it is greater than its neighbors $r(x-d)$ and $r(x+d)$ by a threshold $T$

$$
\left\{\begin{array}{l}
r(x)-r(x-d)>T \\
r(x)-r(x+d)>T
\end{array}\right.
$$

where $d$ is a parameter concerning the width of the vessel to be detected and $T$ is a threshold to evaluate the vessel points.

For (4), it is necessary to determine the parameters $d$ and $T$. These two parameters are not independent. In practice, we use a matched filter $g(x)$ with standard deviation (std) $\sigma$ to detect the vessels whose Gaussian-shaped cross sections have a std around $\sigma$. Thus, we can set

$$
d=c_{d} \sigma
$$

where $c_{d}$ is a constant. As can be seen in Fig. 3(d), a large $c_{d}$ is better able to discriminate the two types of structures. However, if $c_{d}$ is too large, there will be interference between neighboring vessels. If $c_{d}$ is too small, the width of the line will be underestimated. In our experiments, we empirically found that a $c_{d}$ setting of around 2 struck a good balance between detecting the whole line and reducing the interference from neighboring vessels.

Suppose the std of the Gaussian-shaped vessel cross section $f(x)$ to be detected by $g(x)$ is also $\sigma$, then the filtering output $r(x)$ will still be a Gaussian function, and its std will be $\sigma_{r}=$ $\sqrt{\sigma^{2}+\sigma^{2}}=\sqrt{2} \sigma$. Denote by $x_{0}$ the peak point of $r(x)$. It can be easily demonstrated that

$$
\begin{aligned}
r\left(x_{0}\right)-r\left(x_{0}-d\right) & =r\left(x_{0}\right)-r\left(x_{0}+d\right) \\
& =\frac{1}{\sqrt{2 \pi} \sigma_{r}}\left(1-\exp \left(-\frac{d^{2}}{2 \sigma_{r}^{2}}\right)\right) \\
& =\frac{1}{2 \sqrt{\pi} \sigma}\left(1-\exp \left(-\frac{d^{2}}{4 \sigma^{2}}\right)\right) .
\end{aligned}
$$

Let $\Gamma=1 / 2 \sqrt{\pi} \sigma\left(1-\exp \left(-d^{2} / 4 \sigma^{2}\right)\right)$. For a point $x_{1}$ in the neighborhood of $x_{0}$, we can see that $r\left(x_{1}\right)-r\left(x_{1}-d\right)<\Gamma$ and $r\left(x_{1}\right)-r\left(x_{1}+d\right)<\Gamma$. Thus, to detect point $x_{0}$ and its neighbors, which are considered vessel points, we set the threshold $T$ in (4) as

$$
T=c_{T} \Gamma
$$

where $c_{T}$ is a constant and $c_{T}<1$.

Better segmentation results can be produced by adopting the double-thresholding strategy as in [18]. We first set a high threshold $T_{h}=c_{T} \Gamma$ and a low threshold $T_{l}=0.5 T_{h}$, and use them to obtain two vessel maps $V_{l}$ and $V_{h}$. To analyze the characteristics and determination of $c_{T}$, we manually cut many cross sections of the filter responses, normalize the response magnitude into $[0,1]$, and then approximate them using $c(x)=\exp \left(-x^{2} / \sigma^{2}\right)$, for $|x| \leq 3 \sigma$. By varying the values of $c_{T}$, it is possible to calculate different true positives and false positives. We found experimentally that the acceptable accuracy $(>0.9)$ can be obtained by setting $0.5<c_{T}<0.8$. The final vessel map is formed by selecting vessels in $V_{l}$ that link to the vessels in $V_{h}$.

A matched filter with scale $\sigma$ is able to detect the vessels that have a scale around $\sigma$. However, the widths of vessels in a retinal image vary quite a lot, and so it can be used to apply multiple filters $g(x, y)$ at multiple scales. In this paper, we use 


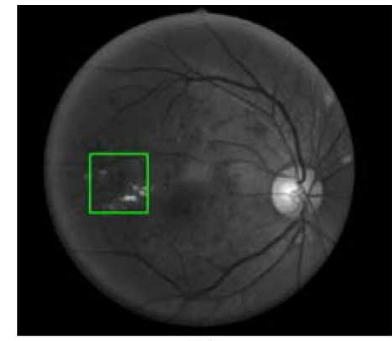

(a)

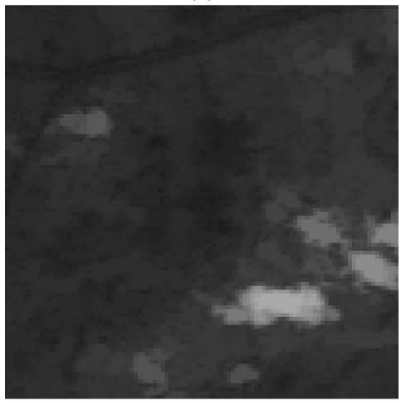

(e)

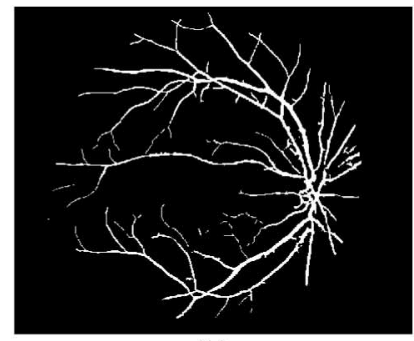

(b)

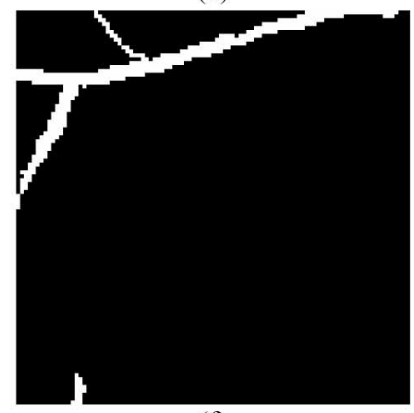

(f)

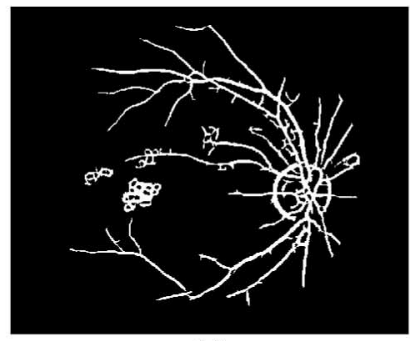

(c)

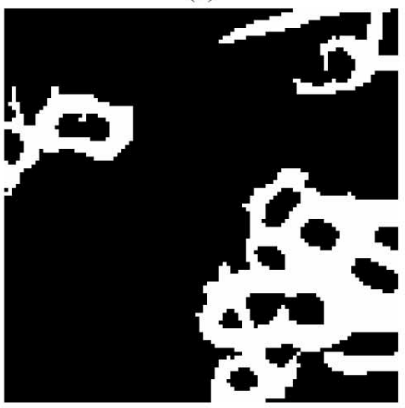

(g)

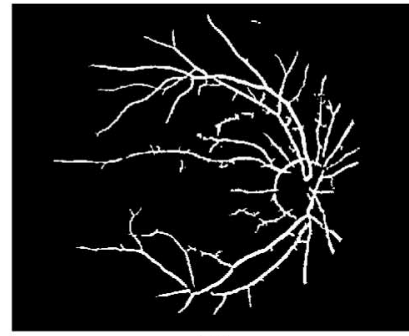

(d)

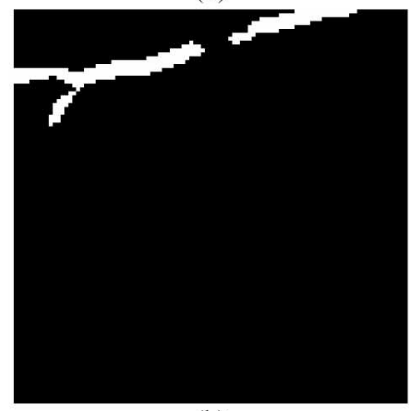

(h)

Fig. 4. (a) Original NPDR image in the ZUEYE database. (b) Hand-labeled ground truth. (c) Extracted vessel map by the scheme in [5]: ACC =0.9449, $\mathrm{TPR}=0.7816, \mathrm{FPR}=0.0373$. (d) Extracted vessel map by the proposed method: ACC $=0.9506$, TPR $=0.7915$, FPR $=0.0320$. (e) $-(\mathrm{h})$ Zoom-in images of (a)-(d). Note that the false vessel detection caused by strong edges of lesions in ( $\mathrm{g}$ ) is reduced greatly in (h).

two filters, ${ }^{1} g_{1}$ with std $\sigma_{1}$ and $g_{2}$ with std $\sigma_{2}$, to obtain two vessel maps $V_{1}$ and $V_{2}$. By fusing the two maps with the logical OR operation, it is possible to construct a more robust vessel map. Note that in order to output meaningful results and avoid generating too many noise responses, the scale $\sigma_{1}$ should not be too small. In the experiment, we use $\sigma_{1}=1$ to find small vessels. To detect large vessels, we set $\sigma_{2}=1.7$ for the ZUEYE database and $\sigma_{2}=2$ for the STARE database (refer to Section IV).

\section{EXPERIMENTAL RESULTS}

The color fundus images were used in the experiments and the green channel was used for vessel extraction. The indexes used to quantitatively measure the performance of different algorithms include: 1) detection accuracy (ACC); 2) the corresponding true positive rate (TPR); and 3 ) the false positive rate (FPR) at that accuracy. The ACC is defined as the ratio of the total number of correctly classified pixels (the vessel pixels classified as vessel pixels and the nonvessel pixels classified as nonvessel pixels) to the number of pixels inside field of view (FOV); the TPR is defined as the ratio of the number of correctly classified vessel pixels to the number of total vessel pixels in the ground truth; the FPR is defined as the ratio of the number of nonvessel pixels inside FOV but classified as vessel pixels to the number of nonvessel pixels inside FOV in the ground truth.

We first evaluated the performance of the proposed scheme on screening PDR by applying it to the ZUEYE database, which consists of retinal images from DR patients. ${ }^{2}$ The color fundus images were captured by using Kowa VK-3 fundus camera at

\footnotetext{
${ }^{1}$ More than two scales can be used to detect the vessels, but based on our experiments, using three or more scales will garner only a small improvement that may not be justified by the additional computational expense.

${ }^{2}$ The images were collected from the patients of the Ophthalmologic Department, the First Affiliated Hospital of Zhengzhou University, Henan Province,
}

TABLE I

VESSEL EXTRACTION RESULTS OF THE ZUEYE DATABASE

\begin{tabular}{cccc}
\hline \hline Method & Accuracy & TPR & FPR \\
\hline Hoover [5] & 0.9380 & 0.7462 & 0.0408 \\
Proposed method & 0.9536 & 0.7954 & 0.0311 \\
\hline \hline
\end{tabular}

$45^{\circ} \mathrm{FOV}$ and were stored in a 24-bit digital format. The resolution of the images is $800 \times 608$. There are 15 retinal images with DR in ZUEYE, including 10 NPDR and 5 PDR. Figs. 4 and 5 each present an example of vessel extraction using the proposed method on an NPDR and a PDR retinal image in ZUEYE. The improved matched filter method developed in [5] is used for comparison. The experimental settings were $\sigma_{1}=1$ to extract small vessels and $\sigma_{2}=1.7$ to extract large vessels. By varying the values of $T_{h}$, different ACC, TPR, and FPR values can be obtained. Table I lists the best averaging accuracies for the two methods.

Fig. 4(a) shows an NPDR retinal image. Fig. 4(b) is the handlabeled ground truth of the vessel map, and Fig. 4(c) and (d) are the extracted vessel maps by the improved matched filter [5] and the proposed method. We see that the proposed method can eliminate most of the false detections of vessels caused by the strong edges of bright lesions. Fig. 4(e)-(h) shows the cropped and zoomed images.

Fig. 5(a) shows a PDR retinal image and Fig. 5(b) is the hand-labeled ground truth of vessel map. Fig. 5(c) and (d) are the extracted vessel maps using the scheme [5] and the proposed method. We see that the proposed method can detect most of the neovasculars (new vessels) and eliminate most of the false detection of vessels caused by the strong edges of bright lesions. We crop and zoom-in part of the images, and show them in Fig. 5(e)-(h). It is seen that the false vessels caused by bright

China. The ground truth maps of vessels were manually labeled by the experts from that hospital. 


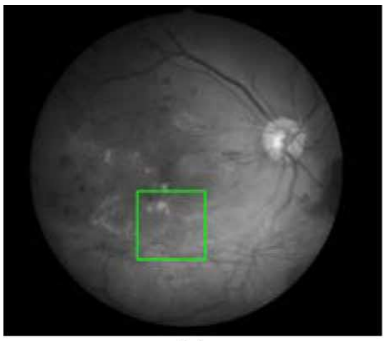

(a)

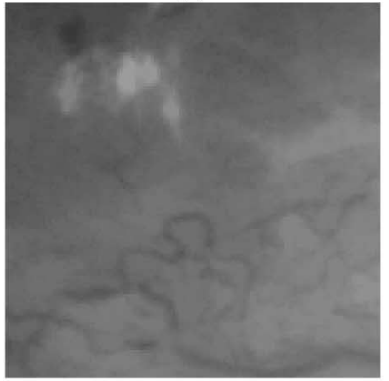

(e)

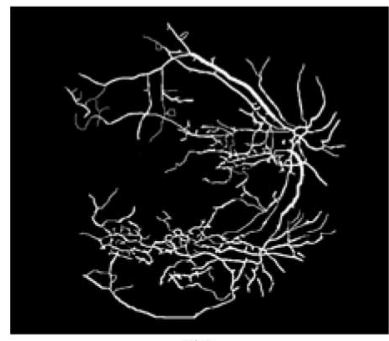

(b)

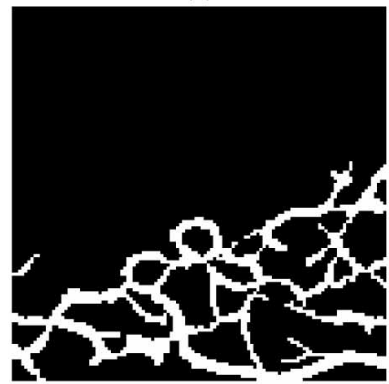

(f)

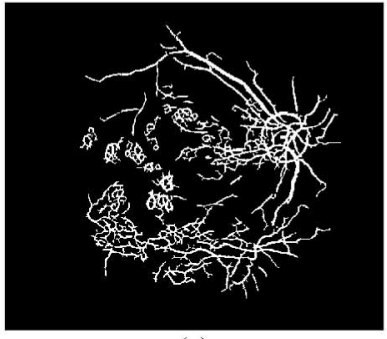

(c)

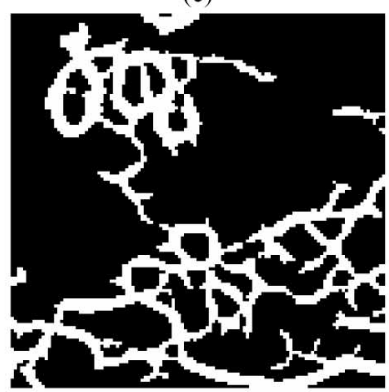

(g)

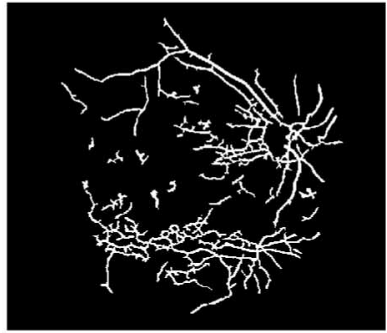

(d)

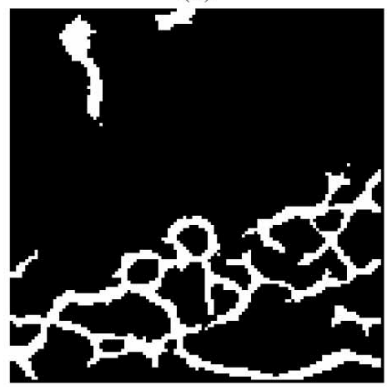

(h)

Fig. 5. (a) Original PDR image in the ZUEYE database. (b) Hand-labeled ground truth. (c) Extracted vessel map by the scheme in [5]: ACC $=0.9243$, TPR $=$ 0.6754, FPR $=0.0479$. (d) Extracted vessel map by the proposed method: ACC $=0.9502$, FPR $=0.0324$, TPR $=0.7959$. (e) $-(\mathrm{h})$ Zoom-in images of (a) $-(\mathrm{d})$. Note that the false vessel detection caused by strong edges of lesions in (e) is reduced greatly in (h), and most of the neovasculars are extracted by the proposed method.

lesions (top left) in Fig. 5(g) are reduced greatly in Fig. 5(h), with the abnormal fine neovascular structures (bottom) being well detected.

Comparing Fig. 4(d) with Fig. 5(d), it is possible to distinguish PDR from NPDR because the PDR image tends to have more vessels in a local window and the neovasculars tend to have large curvature [21], [22]. As shown in Fig. 5(d), most of the neovasculars were extracted by our method and few false vessel pixels were detected. However, as shown in Fig. 4(c), by using Hoover's method, the edges of lesions can be misclassified as neovasculars because they have large local densities and large curvatures. Therefore, the NPDR in Fig. 4(c) may be incorrectly classified as PDR.

We also evaluated the proposed scheme on the publicly available STARE database [4], [5]. The STARE database consists of retinal images captured by the TopCon TRV-50 fundus camera at $35^{\circ} \mathrm{FOV}$. The images were digitized at 24 bits with a spatial resolution of $700 \times 605$ pixels. There are 20 images available for use in vessel detection. Ten of them are from healthy ocular fundus and the other ten images exhibit pathologies. All the 20 images in the SATRE database were used in the experiment. The state-of-the-art schemes [5] and [8] were used for comparison. Table II lists the results of ACC, TPR, and FPR, and Table III compares the running time by different methods.

The results of [8] in Tables II and III are copied from the original paper, in which different color spaces were used for vessel extraction, and it was found that the $\alpha$ channel gives the best result. We copied the best result for comparison. For the results of [5] shown in Figs. 6 and 7, and Tables II and III, we downloaded the segmented images on the associated Web site and calculated the quantitative assessments using these images. The hand-labeled images by the first observer (labels-ah) were
TABLE II

VESSEL EXTRACTION RESULTS OF THE STARE DATABASE

\begin{tabular}{|c|c|c|c|}
\hline Method & Accuracy & $\begin{array}{l}\text { TPR } \\
\end{array}$ & FPR \\
\hline \multicolumn{4}{|c|}{ Normal cases } \\
\hline $2^{\text {nd }}$ Human observer & 0.9283 & 0.8252 & 0.0456 \\
\hline Hoover [5] & 0.9324 & 0.6736 & 0.0528 \\
\hline Mendonça [8] & 0.9531 & 0.7366 & 0.0178 \\
\hline Proposed method & 0.9497 & 0.6611 & 0.0152 \\
\hline \multicolumn{4}{|c|}{ Pathological cases } \\
\hline $2^{\text {nd }}$ Human observer & 0.9425 & 0.8252 & 0.0456 \\
\hline Hoover [5] & 0.9211 & 0.6736 & 0.0528 \\
\hline Mendonça [8] & 0.9426 & 0.6801 & 0.0306 \\
\hline Proposed method & 0.9416 & 0.7286 & 0.0372 \\
\hline
\end{tabular}

TABLE III

RUNNING TIME PER IMAGE IN THE STARE DATABASE

\begin{tabular}{ccc}
\hline \hline Method & System Environment & Running Time \\
\hline Hoover [5] & P-III 1.5GHz, 512 Mb RAM, Windows executable & 0.5 minute \\
Mendonça [8] & P-IV 3.2GHz, 960 Mb RAM, Matlab & 3 minutes \\
Proposed method & P-III 1.5GHz, 512 Mb RAM, Matlab & 0.5 minute \\
\hline \hline
\end{tabular}

used as ground truth. We set $\sigma_{1}=1$ to extract small vessels and $\sigma_{2}=2$ to extract large vessels. Table II lists the best averaging accuracies for normal images and pathological images.

The scheme in [5] tries to eliminate the false positives caused by lesions through threshold probing and the scheme in [8] tries to eliminate the false positives caused by lesions through combing the centerline detection and a modified top-hat operation. Fig. 6(a) shows a pathological image in the STARE database [5]; Fig. 6(b) shows the manually labeled ground truth by the first observer (labels-ah); Fig. 6(c) shows the extracted vessel image by [5]; and Fig. 6(d) shows the vessel map by using the proposed method. We see that the proposed method can detect more of small vessels at a low FPR. Most of the false vessel detections in [5] are eliminated by the proposed method. This is very important because the edges of bright lesions have large curvatures, as shown in Fig. 6(c), and so they can be easily misclassified as neovasculars 


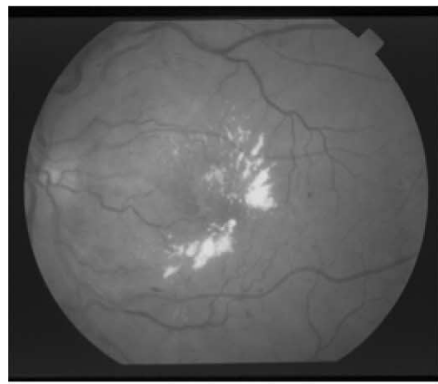

(a)

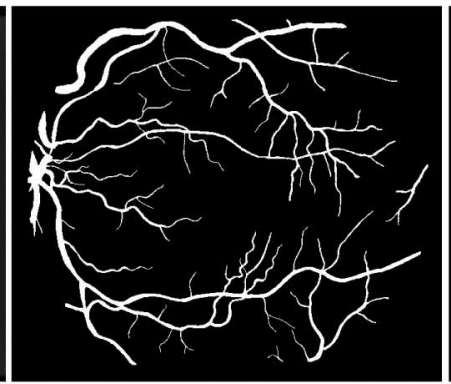

(b)

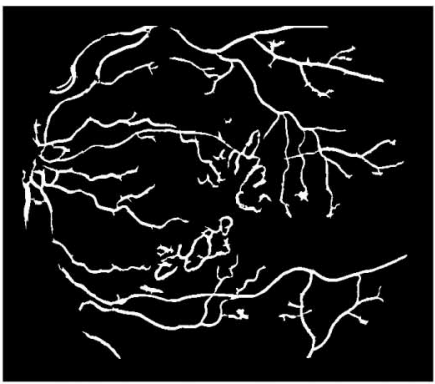

(c)

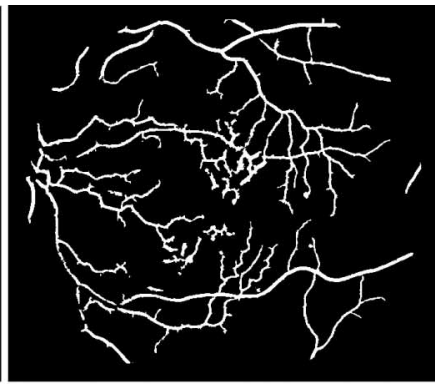

(d)

Fig. 6. (a) Pathological image in the STARE database. (b) Hand-labeled ground truth. (c) Extracted vessel map by the scheme [5]: ACC $=0.9198$, TPR $=$ $0.5937, \mathrm{FPR}=0.0402$. (d) Extracted vessel map by the proposed method: $\mathrm{ACC}=0.9302, \mathrm{TPR}=0.6619, \mathrm{FPR}=0.0368$.

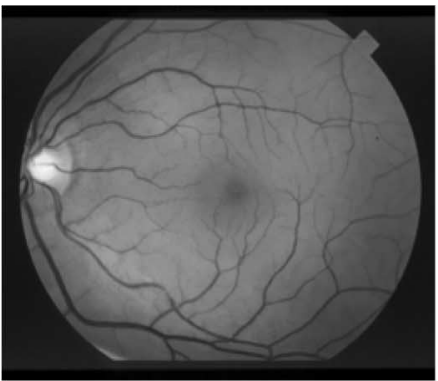

(a)

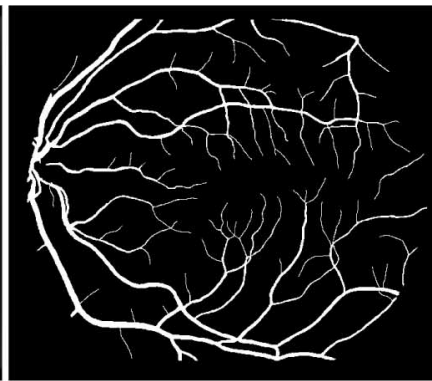

(b)

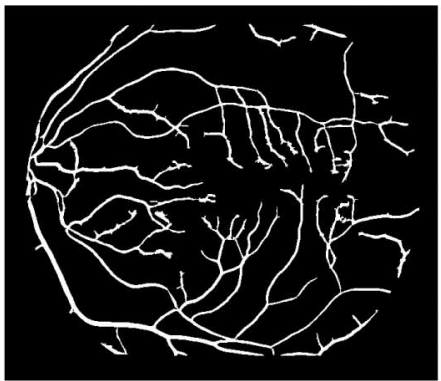

(c)

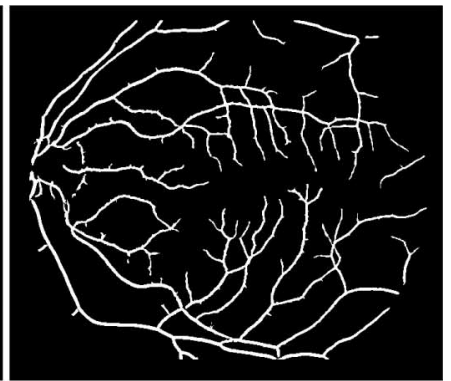

(d)

Fig. 7. (a) Normal image in the STARE database. (b) Hand-labeled ground truth. (c) Extracted vessel map by the scheme in [5]: ACC $=0.9320$, TPR $=0.6992$, $\mathrm{FPR}=0.0393$. (d) Extracted vessel map by the proposed method: ACC $=0.9487, \mathrm{TPR}=0.7625, \mathrm{FPR}=0.0283$.

Fig. 7(a) shows a normal image in the STARE database [5]; Fig. 7(b) shows the manually labeled ground truth by the first observer (labels-ah); Fig. 7(c) shows the extracted vessel image of [5]; and Fig. 7(d) shows the vessel map by using the proposed method. We see that the proposed method can detect more small vessels and has lesser false detections than [5].

From Tables II and III, we can see that the proposed method is very competitive with the state-of-the-art methods [5] and [8]. It achieves the highest TPR among the three schemes for the pathological images and the FPR is very low. Meanwhile, it can be seen that our algorithm has relatively low computational complexity. (Our method has been evaluated, prior to optimization, using Matlab modules. Although in Table III, the method [5] has relatively the same computational performance as ours, we believe that our algorithm will have improved performance when implemented in Windows executable modules.)

\section{CONCLUSION}

In this paper, we presented a modified matched filter approach for the detection of blood vessels in retinal images while suppressing the nonvessel edge structures. The modified matched filter avoids responding to nonline edges by applying local double-sided thresholding. Experiments on retinal images demonstrate that the proposed method can detect neovascular nets and eliminate many nonvessel edges caused by bright lesions, which is a desirable property in PDR screening because the large curved edges of bright lesions are prone to misclassification as neovascular nets.

\section{ACKNOWLEDGMENT}

The authors sincerely thank the associate editor and the anonymous reviewers for their constructive comments.

\section{REFERENCES}

[1] J. J. Staal, M. D. Abràmoff, M. Niemeijer, M. A. Viergever, and B. van Ginneken, "Ridge based vessel segmentation in color images of the retina," IEEE Trans. Med. Imag., vol. 23, no. 4, pp. 501-509, Apr. 2004.

[2] J. V. B. Soares, J. J. G. Leandro, R. M. Cesar, Jr., H. F. Jelinek, and M. J. Cree, "Retinal vessel segmentation using the 2-D Gabor wavelet and supervised classification," IEEE Trans. Med. Imag., vol. 25, no. 9, pp. 1214-1222, Sep. 2006

[3] G. G. Yen and W.-F. Leong, "A sorting system for hierarchical grading of diabetic fundus images: A preliminary study," IEEE Trans. Inf. Technol. Biomed., vol. 12, no. 1, pp. 118-130, Jan. 2008.

[4] S. Chaudhuri, S. Chatterjee, N. Katz, M. Nelson, and M. Goldbaum, "Detection of blood vessels in retinal images using two-dimensional matched filters," IEEE Trans. Med. Imag., vol. 8, no. 3, pp. 263-269, Sep. 1989.

[5] A. Hoover, V. Kouznetsova, and M. Goldbaum, "Locating blood vessels in retinal images by piecewise threshold probing of a matched filter response," IEEE Trans. Med. Imag., vol. 19, no. 3, pp. 203-210, Mar. 2000.

[6] D. Wu, M. Zhang, J. C. Liu, and W. Bauman, "On the adaptive detection of blood vessels in retinal images," IEEE Trans. Biomed. Eng., vol. 53, no. 2, pp. 341-343, Feb. 2006.

[7] F. Zana and J.-C. Klein, "Segmentation of vessel-like patterns using mathematical morphology and curvature evaluation," IEEE Trans. Image Process., vol. 10, no. 7, pp. 1010-1019, Jul. 2001.

[8] A. M. Mendonça and A. Campilho, "Segmentation of retinal blood vessels by combining the detection of centerlines and morphological reconstruction," IEEE Trans. Med. Imag., vol. 25, no. 9, pp. 1200-1213, Sep. 2006.

[9] X. Jiang and D. Mojon, "Adaptive local thresholding by verification based multithreshold probing with application to vessel detection in retinal images," IEEE Trans. Pattern Anal. Mach. Intell, vol. 25, no. 1, pp. 131-137, Jan. 2003. 
[10] F. Zana and J.-C. Klein, "Segmentation of vessel-like patterns using mathematical morphology and curvature evaluation," IEEE Trans. Image Process., vol. 10, no. 7, pp. 1010-1019, Jul. 2001.

[11] M. E. Martínez-Pérez, A. D. Hughes, A. V. Stanton, S. A. Thom, A. A. Bharath, and K. H. Parker, "Retinal blood vessel segmentation by means of scale-space analysis and region growing," in Proc. 2nd MICCAI, 1999, pp. 90-97.

[12] M. E. Martínez-Pérez, A. D. Hughes, and S. A. Thom, "Segmentation of blood vessels from red-free and fluorescein retinal images," Med. Image Anal., vol. 11, no. 1, pp. 47-61, Feb. 2007.

[13] Y. Tolias and S. Panas, "A fuzzy vessel tracking algorithm for retinal images based on fuzzy clustering," IEEE Trans. Med. Imag., vol. 17, no. 2, pp. 263-273, Apr. 1998.

[14] A. Can, H. Shen, J. N. Turner, H. L. Tanenbaum, and B. Roysam, "Rapid automated tracing and feature extraction from retinal fundus images using direct exploratory algorithms," IEEE Trans. Inf. Technol. Biomed., vol. 3, no. 2, pp. 125-138, Jun. 1999.

[15] I. Liu and Y. Sun, "Recursive tracking of vascular networks in angiograms based on the detection-deletion scheme," IEEE Trans. Med. Imag., vol. 12, no. 2, pp. 334-341, Jun. 1993.

[16] L. A. Iverson and S. W. Zucker, "Logical/linear operators for image curves," IEEE Trans. Pattern Anal. Mach. Intell., vol. 17, no. 10, pp. $982-$ 996, Oct. 1995.

[17] C. Steger, "An unbiased detector of curvilinear structures," IEEE Trans. Pattern Anal. Mach. Intell., vol. 20, no. 2, pp. 113-125, Feb. 1998.

[18] J. Canny, "A computational approach to edge detection," IEEE Trans. Pattern Recognit. Mach. Intell., vol. PAMI-8, no. 6, pp. 679-698, Nov. 1986.

[19] R. C. Gonzales and R. E. Woods, Digital Image Processing. Reading, MA: Addison-Wesley, 1993.

[20] T. M. Koller, G. Gerig, G. Székely, and D. Dettwiler, "Multiscale detection of curvilinear structures in 2-D and 3-D image data," in Proc. 5th Int. Conf. Comput. Vision. Los Alamitos, CA: IEEE Comput. Soc. Press, 1995, pp. 864-869.

[21] E. J. Sussman, W. G. Tsiaras, and K. A. Soper, "Diagnosis of diabetic eye disease," J. Amer. Med. Assoc., vol. 247, no. 23, pp. 3231-3234, Jun. 1982.

[22] C. P. Wilkinson, F. L. Ferris, R. E. Klein, P. P. Lee, C. D. Agardh, M. Davis, D. Dills, A. Kampik, R. Pararajasegaram, and J. T. Verdaguer, "Proposed international clinical diabetic retinopathy and diabetic macular edema disease severity scales," Ophthalmology, vol. 110, no. 9, pp. 1677-1682, Sep. 2003.

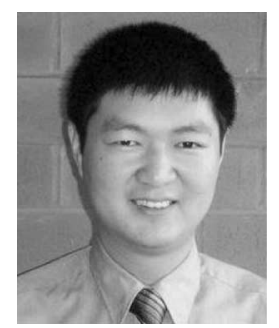

Lei Zhang (M'04) received the B.S. degree from Shenyang Institute of Aeronautical Engineering, Shenyang, China, in 1995, and the M.Sc. and Ph.D. degrees in electrical and engineering from Northwestern Polytechnical University, Xi'an, China, in 1998 and 2001, respectively.

From 2001 to 2002, he was a Research Associate in the Department of Computing, the Hong Kong Polytechnic University, Kowloon, Hong Kong, where he has been an Assistant Professor since January 2006. From January 2003 to January 2006, he was a Postdoctoral Fellow in the Department of Electrical and Computer Engineering, McMaster University, Canada. His current research interests include image and video processing, biometrics, pattern recognition, multisensor data fusion, and optimal estimation theory.

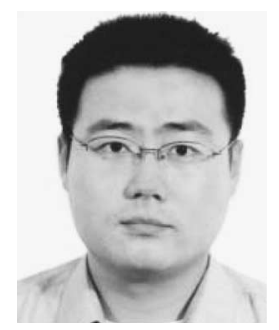

Qin $\mathbf{L i}$ received the B.Eng. degree in computer science from China University of Geoscience, Wuhan, China, in 2001, and the M.Sc. degree (with distinction) in computing from the University of NorthUmbria at Newcastle, Newcastle, U.K., in 2003. He is currently working toward the Ph.D. degree at the Biometric Research Center, Department of Computing, the Hong Kong Polytechnic University, Kowloon, Hong Kong.

He is a Lecturer at the Henan Provincial Key Laboratory on Information Networks, Zhengzhou University, Zhengzhou, China. His current research interests include medical image analysis, biometrics, image processing, and pattern recognition.

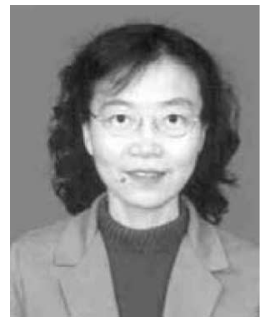

Jane You (M'95) received the B.Eng. degree in electronic engineering from Xi' an Jiaotong University, Xi' an, China, in 1986, and the Ph.D. degree in computer science from La Trobe University, Melbourne, Vic., Australia, in 1992.

From 1993 to 1995, she was a Lecturer in the School of Computing and Information Science, University of South Australia. From 1996 to 2001, she was with the School of Computing and Information Technology, Griffith University, Australia, where she was a Lecturer (1996-1998) and later a Senior Lecturer (1999-2001). She is currently an Associate Professor in the Department of Computing, the Hong Kong Polytechnic University, Kowloon, Hong Kong. Her current research interests include visual information retrieval, image processing, pattern recognition, multimedia systems, biometrics computing, and data mining. She is the author or coauthor of more than 140 research papers published as journal articles, book chapters, and conference publications.

Dr. You was awarded a French Foreign Ministry International Fellowship in 1993.

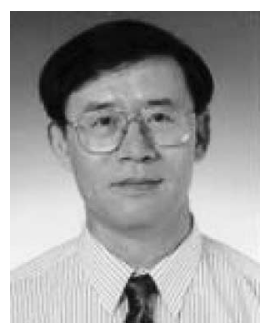

David Zhang (F'08) received the Graduate degree in computer science from Peking University, Beijing, China, the M.Sc. degree in computer science in 1982 and the Ph.D. degree in 1985 from the Harbin Institute of Technology (HIT), Harbin, China, and the second $\mathrm{Ph} . \mathrm{D}$. degree in electrical and computer engineering from the University of Waterloo, Waterloo, ON, Canada, in 1994

From 1986 to 1988, he was a Postdoctoral Fellow at Tsinghua University, Beijing. He then became an Associate Professor at the Academia Sinica, Beijing. He is currently a Chair Professor at the Hong Kong Polytechnic University, Kowloon, Hong Kong, where he is the Founding Director of the Biometrics Technology Centre (UGC/CRC) supported by the Hong Kong SAR Government in 1998. He is also a Visiting Chair Professor at Tsinghua University, and an Adjunct Professor at Shanghai Jiao Tong University, Shanghai; Harbin Institute of Technology; and the University of Waterloo. He is the Founder and the Editor-in-Chief of the International Journal of Image and Graphics (IJIG) and a Book Editor of the Springer International Series on Biometrics (KISB). $\mathrm{He}$ is an Associate Editor of more than ten international journals including Pattern Recognition. He is the author or coauthor of more than ten books and 190 journal papers.

Prof. Zhang is an organizer of the International Conference on Biometrics Authentication (ICBA). He is an Associate Editor of the IEEE TRANSACTIONS ON SYSTEMS, MAN, AND CYBERNETICS-PART A: SYSTEMS AND HUMANS and IEEE TRANSACTIONS ON SYSTEMS, MAN, AND CYBERNETICS-PART C: APPLICATIONS AND REviEws. He is also the Technical Committee Chair of the IEEE Computational Intelligence Society (CIS). He is a Croucher Senior Research Fellow, a Distinguished Speaker of the IEEE Computer Society, and a Fellow of the International Association of Pattern Recognition (IAPR). 